\title{
Periodic arrangements of chiral scatterers providing negative refractive index bi-isotropic media
}

\author{
L. Jelinek, ${ }^{1, *}$ R. Marqués, ${ }^{1, \dagger}$ F. Mesa, ${ }^{2, \pitchfork}$ and J. D. Baena ${ }^{1,3, \$}$ \\ ${ }^{1}$ Departamento de Electrónica y Electromagnetismo, Universidad de Sevilla, 41012-Sevilla, Spain \\ ${ }^{2}$ Departamento de Física Aplicada 1, Universidad de Sevilla, 41012-Sevilla, Spain \\ ${ }^{3}$ Departamento de Física, Universidad Nacional de Colombia, Bogotá, Colombia
}

(Received 14 April 2008; published 14 May 2008)

\begin{abstract}
The main goal of this contribution is to show that periodic arrangements of chiral scatterers can be the basis for the development of three dimensional and isotropic negative refractive index artificial media. Three dimensionality and isotropy are key issues in this context since only three dimensional structures can be properly viewed as a "medium," and only in isotropic media the refractive index is unambiguously defined. The proposed arrangements are cubic lattices of chiral split ring resonators conveniently designed to yield an isotropic behavior. The reported structures are shown to provide a significant frequency band of negative refraction with good matching to free space.
\end{abstract}

DOI: 10.1103/PhysRevB.77.205110 PACS number(s): 41.20.Jb, 42.70.Qs, 78.20.Ci, 78.20.Ek

\section{INTRODUCTION}

Isotropic negative refractive index (NRI) media were first proposed and analyzed by Veselago in $1968,{ }^{1}$ but its practical implementation was not envisaged until recently, after the results of Smith et al. ${ }^{2}$ It should be pointed out that the refraction index can only be unambiguously defined in three dimensional isotropic media and that most reported practical realizations of media exhibiting negative refraction fall into the category of indefinite media ${ }^{3}$ rather than into the category of isotropic NRI or "left-handed"1 media. Actually, the development of an isotropic NRI medium is still a challenging issue despite the diverse efforts in this direction. ${ }^{4-7}$ Overcoming this challenge is of interest not only from a theoretical standpoint but also from a practical one: isotropic and bi-isotropic NRI media will be useful for designing three dimensional planar focusing devices, ${ }^{1,8}$ subdiffraction imaging devices, ${ }^{9}$ and other applications.

Chiral scatterers are an attractive alternative for the design of NRI artificial media because of their ability for providing simultaneously negative permittivity and permeability. To the best of our knowledge, the first proposals in this direction were made by Tretyakov and co-workers. ${ }^{10,11}$ Other proposals aimed to take advantage of chirality for NRI media design were subsequently reported. ${ }^{12,13}$ All these proposals either neglect the issue of isotropy or propose random arrangements to address it. However, random arrangements, although conceptually appealing, are not easily reproducible and do not show a well defined electromagnetic response, mainly if the size of inclusions is not sufficiently small compared to wavelength (as usual in most artificial media). In fact, this last feature could be one of the main reasons why isotropic NRI artificial media made from chiral inclusions have not been developed further, remaining only as a theoretical possibility. By contrast, periodic arrangements are reproducible, and the eigenmodes of the structure can be unambiguously identified by using Bloch-Floquet theorem. Starting from these considerations, this paper proposes a way to the experimental realization of bi-isotropic and isotropic NRI artificial media using periodic arrangements of chiral scatterers. The paper is organized as follows. First, the basic structure is proposed and an approximate analytical theory based on a generalization of Lorentz homogenization procedure is developed. This analysis directly provides the appropriate design parameters. Then, rigorous full-wave electromagnetic simulations are carried out in order to identify the eigenmodes of the structure and to validate the analytical theory. These simulations are also useful in order to identify the effects of spatial dispersion, which are not taken into account by the analytical theory. As will be shown, these effects are not relevant in the frequency band of interest. Next, the impedance matching to free space of the proposed structure is studied. Finally, the possibility of creating a racemic configuration is discussed.

\section{BALANCED CHIRAL BI-ISOTROPIC MEDIA}

Before the analysis of any specific structure, some key properties of bi-isotropic chiral media will be recalled. Biisotropic media can be described by the following linear constitutive relations: ${ }^{14}$

$$
\begin{gathered}
\mathbf{D}=\varepsilon_{0}\left(1+\chi_{e}\right) \mathbf{E}+j \sqrt{\varepsilon_{0} \mu_{0}} \kappa \mathbf{H}, \\
\mathbf{B}=-j \sqrt{\varepsilon_{0} \mu_{0}} \kappa \mathbf{E}+\mu_{0}\left(1+\chi_{m}\right) \mathbf{H},
\end{gathered}
$$

where $\chi_{e}, \chi_{m}$, and $\kappa$ are the electric, magnetic, and cross susceptibilities, respectively, which are real quantities for lossless media. Assuming an $\exp (j \omega t)$ time dependence, the eigenwaves propagating through these media are right- and left-circularly polarized plane waves whose dispersion relation is given by ${ }^{14}$

$$
k^{ \pm}=k_{0}\left(\sqrt{\mu_{r} \varepsilon_{r}} \pm \kappa\right),
$$

where $k_{0}=\omega \sqrt{\varepsilon_{0} \mu_{0}}$ and $\varepsilon_{r}=\left(1+\chi_{e}\right), \mu_{r}=\left(1+\chi_{m}\right)$. In order to reduce the forbidden bands of propagation coming from complex values of $k^{ \pm}$, it is desirable that $\chi_{e}(\omega) \simeq \chi_{m}(\omega)$ so that $\mu_{r}$ and $\epsilon_{r}$ always have the same sign. It is known ${ }^{11,13}$ that this condition also implies that

$$
\chi_{e}(\omega) \simeq \chi_{m}(\omega) \simeq|\kappa(\omega)| .
$$

The conditions for negative refraction in transparent biisotropic chiral media are also known. ${ }^{15}$ In lossless bi- 


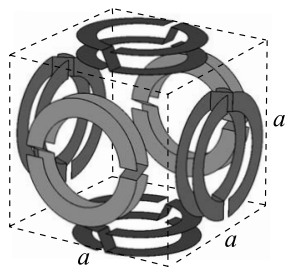

(a)

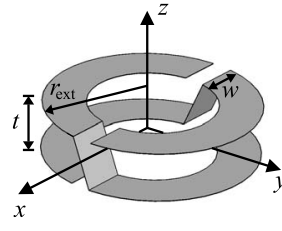

(b)
FIG. 1. (a) Cubic arrangement of chiral SRRs satisfying $T$ group of symmetry (namely, any of the in-body diagonals is a third order rotation symmetry axis) and (b) detail of the chiral SRR design.

isotropic media satisfying the balance condition [Eq. (4)], these conditions reduce to

$$
\chi_{e}<-0.5 \text { and } \chi_{m}<-0.5
$$

or, equivalently, $\varepsilon_{r}<0.5$ and $\mu_{r}<0.5,{ }^{13}$ which is less restrictive than the usual condition for conventional isotropic media $\left(\varepsilon_{r}<0\right.$ and $\left.\mu_{r}<0\right)$. The price to pay for this enhanced bandwidth is that only one of the eigenwaves of Eq. (3) will exhibit negative refraction, while the other will present a positive refractive index. ${ }^{8,13}$

Both eigenwaves have the same wave impedance given by ${ }^{14}$

$$
Z=Z_{0} \sqrt{\frac{\mu_{r}}{\varepsilon_{r}}},
$$

where $Z_{0}=\sqrt{\mu_{0} / \varepsilon_{0}}$ is the free space impedance. However, since the refractive index is different for each eigenmode, the refracted amplitude is not necessarily the same for both eigenmodes. In general, both eigenmodes will be generated at the interface between free space (or other isotropic medium) and a chiral medium, even if the incident wave is circularly polarized. The only exception to this rule is normal incidence.

\section{THEORY AND DESIGN}

In a previous paper, ${ }^{16}$ some of the authors showed that a cubic arrangement of scatterers satisfying the tetrahedral or (in Schoenflies notation) $T$ group of symmetry provides a useful basis for the design of isotropic artificial media. Figure 1(a) shows a cubic arrangement of chiral split ring resonators (SRRs) satisfying this symmetry. It should be noticed that the SRRs on opposite sides of the cube of Fig. 1(a) are identical, which allows for the realization of an artificial medium by means of the periodic repetition of this structure. This artificial medium has a simple cubic lattice of periodicity $a$ (the edge of the cube) and its primitive unit cell contains three chiral SRRs. Note that the proposed chiral SRR is a practical design, feasible to be manufactured by standard planar-circuit fabrication techniques. ${ }^{20}$

An easy way to compute the susceptibilities of the metamaterial can be obtained from a straightforward extension of Lorentz local field theory that takes into account the presence of cross polarizabilities. This simple theory leads to the following equations for the macroscopic polarizations, $\mathbf{P}$ and $\mathbf{M}$, of the metamaterial:

$$
\begin{aligned}
& \mathbf{P}=\frac{1}{V}\left[\left\langle\alpha_{e}\right\rangle\left(\mathbf{E}+\frac{\mathbf{P}}{3 \varepsilon_{0}}\right)+\mu_{0}\left\langle\alpha_{e m}\right\rangle\left(\mathbf{H}+\frac{\mathbf{M}}{3}\right)\right], \\
& \mathbf{M}=\frac{1}{V}\left[\mu_{0}\left\langle\alpha_{m}\right\rangle\left(\mathbf{H}+\frac{\mathbf{M}}{3}\right)-\left\langle\alpha_{e m}\right\rangle\left(\mathbf{E}+\frac{\mathbf{P}}{3 \varepsilon_{0}}\right)\right],
\end{aligned}
$$

where $V=a^{3}$ is the volume of the unit cell and $\left\langle\alpha_{e}\right\rangle,\left\langle\alpha_{e m}\right\rangle$, and $\left\langle\alpha_{m}\right\rangle$ are the average polarizabilities of the unit cell, which are defined as

$$
\begin{gathered}
\mathbf{p}=\left\langle\alpha_{e}\right\rangle \mathbf{E}_{l}+\left\langle\alpha_{e m}\right\rangle \mathbf{B}_{l}, \\
\mathbf{m}=-\left\langle\alpha_{e m}\right\rangle \mathbf{E}_{l}+\left\langle\alpha_{m}\right\rangle \mathbf{B}_{l},
\end{gathered}
$$

where $\mathbf{p}$ and $\mathbf{m}$ are the electric and magnetic dipoles induced at each unit cell, $\mathbf{E}_{l}$ and $\mathbf{B}_{l}$ are the electric and magnetic local fields, and where Onsager symmetries ${ }^{17}$ for the magnetoelectric polarizabilities are explicitly introduced $\left(\left\langle\alpha_{m e}\right\rangle\right.$ $\left.=-\left\langle\alpha_{e m}\right\rangle\right)$. By using Eqs. (7) and (8), the metamaterial susceptibilities are directly obtained from their definitions, $\varepsilon_{0} \chi_{e}=\partial \mathbf{P} / \partial \mathbf{E}$ and $\chi_{m}=\partial \mathbf{M} / \partial \mathbf{H}$, provided that the polarizabilities are known. These polarizabilities can be obtained from the polarizabilities of the chiral SRR. Analytical expressions for the chiral SRR polarizabilities were already reported ${ }^{18}$ and will be here reproduced for completeness,

$$
\begin{gathered}
\alpha_{z z}^{m m}=\frac{\pi^{2} r^{4}}{L} \frac{\omega^{2}}{\omega_{0}^{2}-\omega^{2}+j \omega R / L}, \\
\alpha_{z z}^{e m}= \pm j \frac{2 \pi r^{2} t}{\omega_{0} L}\left(\frac{\omega_{0}}{\omega}\right) \frac{\omega^{2}}{\omega_{0}^{2}-\omega^{2}+j \omega R / L}, \\
\alpha_{z z}^{e e}=\frac{4 t^{2}}{\omega_{0}^{2} L}\left(\frac{\omega_{0}}{\omega}\right)^{2} \frac{\omega^{2}}{\omega_{0}^{2}-\omega^{2}+j \omega R / L}, \\
\alpha_{x x}^{e e}=\alpha_{y y}^{e e}=\varepsilon_{0} \frac{16}{3} r_{\mathrm{ext}}^{3},
\end{gathered}
$$

where $t$ and $r_{\mathrm{ext}}$ are geometrical parameters [see Fig. 1(b)] and $L, R$, and $\omega_{0}$ are the chiral SRR self-inductance, resistance, and frequency of resonance, respectively, which can be analytically obtained from a general procedure already developed by some of the authors. ${ }^{19}$ From Eqs. (11)-(14), the average polarizabilities in Eqs. (9) and (10) are obtained as

$$
\begin{gathered}
\left\langle\alpha_{e}\right\rangle=\alpha_{x x}^{e e}+\alpha_{y y}^{e e}+\alpha_{z z}^{e e}, \\
\left\langle\alpha_{e m}\right\rangle=\alpha_{z z}^{e m}, \\
\left\langle\alpha_{m}\right\rangle=\alpha_{z z}^{m m} .
\end{gathered}
$$

When the balance condition [Eq. (4)] is translated to the unit cell polarizabilities, it becomes

$$
c^{2}\left\langle\alpha_{e}\right\rangle=c\left|\left\langle\alpha_{e m}\right\rangle\right|=\left\langle\alpha_{m}\right\rangle,
$$

where $c$ is the velocity of light. This condition cannot be exactly fulfilled at all frequencies using the polarizabilities from Eqs. (11)-(14) due to the presence of the static electric polarizabilities [Eq. (14)]. However, if the static electric po- 


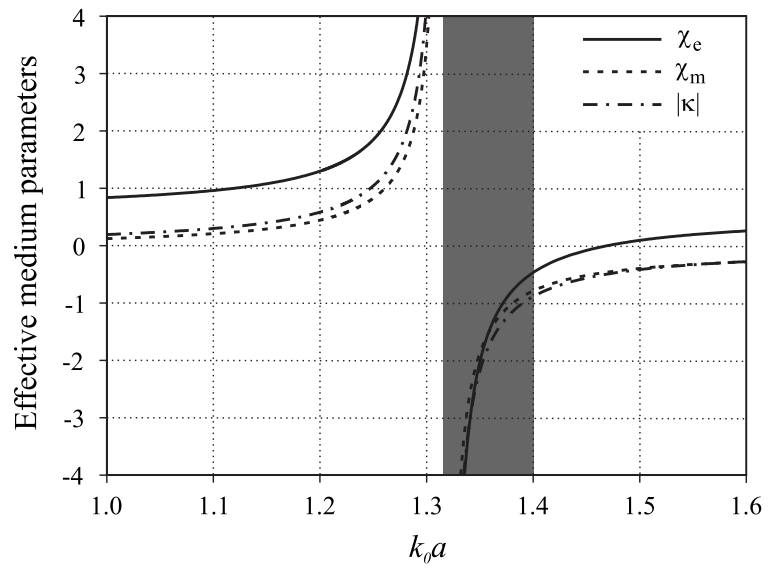

FIG. 2. Theoretical constitutive parameters $\chi_{e}, \chi_{m}$, and $\kappa$ for the metamaterial made of the periodic repetition of the cubic arrangement of Fig. 1(a), forming a simple cubic lattice with periodicity $a$. SRR parameters are $a / r_{\text {ext }}=2.87, w / r_{\text {ext }}=0.4$, and $t / r_{\text {ext }}=0.47$.

larizabilities are neglected (which is a reasonable hypothesis near resonance), the balance condition [Eq. (18)] is approximately satisfied provided that

$$
t \lambda_{0}=(\pi r)^{2}
$$

where $\lambda_{0}$ is the wavelength at resonance and $t$ and $r=r_{\mathrm{ext}}$ $-w / 2$ are structural parameters of the chiral SRR [see Fig. 1(b)]. Although Eq. (19) is only approximate and comes from a simplified homogenization procedure, it will be shown that it is a very good design rule in the frequency band of interest.

In the following, a particular example is analyzed in order to illustrate and validate our analytical predictions. The chosen metamaterial parameters satisfy Eq. (19) and are given in the caption of Fig. 2. The frequency band of left-handed propagation, given by Eq. (5), is marked in gray. It can be seen how inside this region the balance condition [Eq. (4)] is approximately fulfilled. However, outside this region, the static nonresonant polarizabilities [Eq. (14)] dominate and the balance condition is not fulfilled. The theoretical dispersion curves for the eigenwaves of the bi-isotropic metamaterial, given by Eq. (3), are shown in Fig. 3. A frequency band of backward-wave propagation for one of the eigenwaves can be clearly observed (between points marked as 2 and 4), which corresponds to the left-handed frequency band shown in Fig. 2. Since backward-wave propagation is the signature of negative refraction, ${ }^{1}$ this region corresponds to a NRI for the considered eigenmode. Inside this band, there is also a small forbidden band gap (marked as 3; see also the inset) that corresponds to complex values of the propagation constants [Eq. (3)]. This small band gap is due to the aforementioned approximations implicit in Eq. (19). The straight horizontal line at the frequency where the propagation constant of one of the eigenwaves vanishes (corresponding to the condition $\varepsilon_{r} \mu_{r}=\kappa^{2}$ ) represents a fully degenerate longitudinal wave with $\mathbf{k} \times \mathbf{E}=0$ and $\mathbf{k} \times \mathbf{H}=0$.

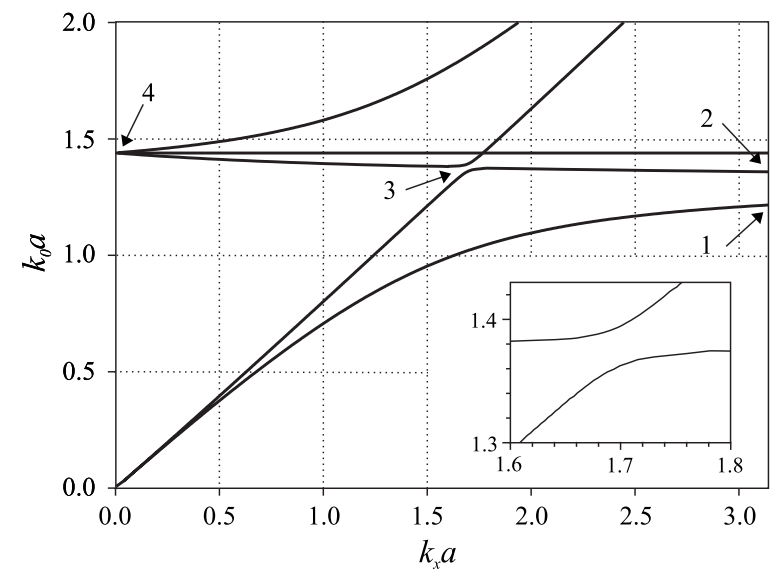

FIG. 3. Theoretical dispersion curves for the eigenwaves of the metamaterial shown in Fig. 2. Dispersion curves are drawn inside the first Brillouin zone of the structure.

\section{ELECTROMAGNETIC SIMULATIONS}

In this section, the previously studied structure is analyzed by means of the commercial electromagnetic simulator CST MICROWAVE STUDIO, giving the band structure shown in Fig. 4. A good qualitative agreement is found between Figs. 3 and 4 . In both figures, a frequency band of backward-wave propagation is observed for one of the eigenwaves. Also, a small frequency stopband appears in Fig. 4 inside this backward-wave passband (because of the approximate balance condition employed) in agreement with the theoretical predictions of Fig. 3. In order to show the isotropic nature of the structure, the dispersion curves along the $\Gamma-X, \Gamma-M$, and $\Gamma-R$ paths are shown in Fig. 5. The isotropy of the dispersion relation for the transverse modes becomes apparent from these curves, except for high values of the propagation constant (namely, close to the border of the Brillouin zone), where spatial dispersion affects the dispersion relation. The longitudinal mode of Fig. 3 also appears in Figs. 4 and 5, although now spatial dispersion destroys degeneracy and isotropy.

The main differences between Figs. 4 and 5 and the theoretical dispersion curves shown in Fig. 3 are observed at

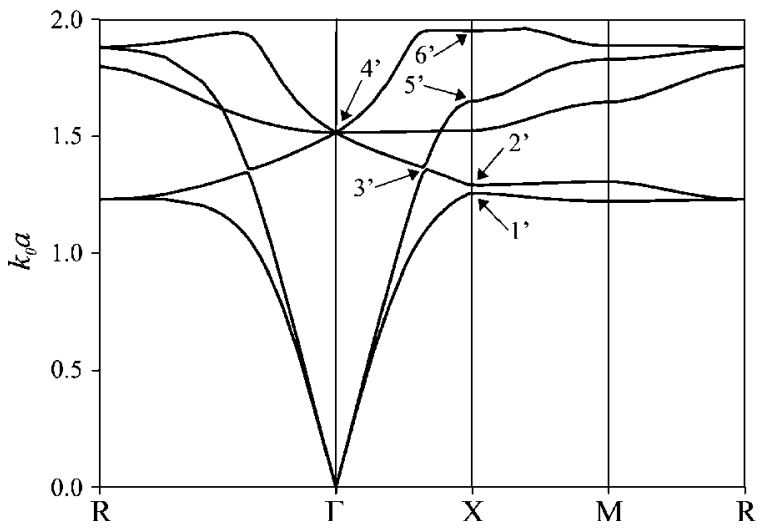

FIG. 4. Band structure and dispersion relation for the metamaterial of Fig. 2 obtained from full-wave simulations along the path $R-\Gamma-X-M-R[\Gamma$ is the center of the $k$-space, $X=(\pi / a, 0,0), M$ $=(\pi / a, \pi / a, 0)$, and $R=(\pi / a, \pi / a, \pi / a)]$. 


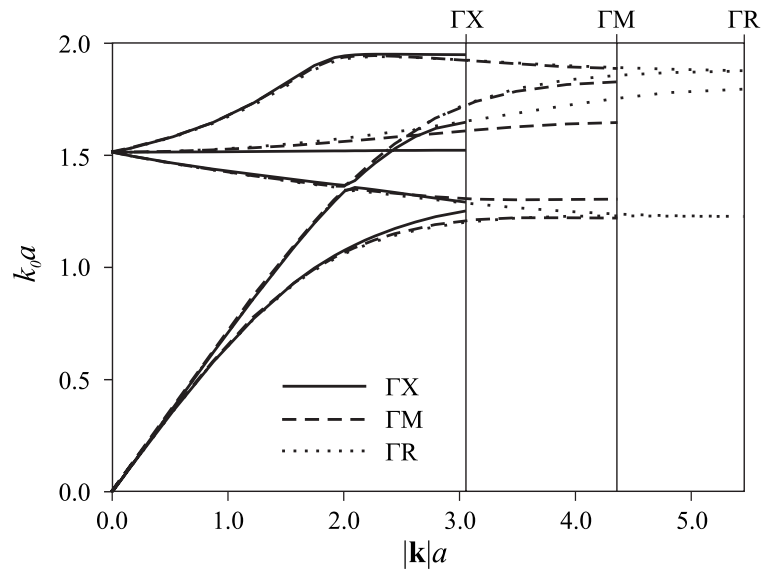

FIG. 5. Dispersion curves along the $\Gamma-X, \Gamma-M$, and $\Gamma-R$ directions for the eigenwaves of the balanced metamaterial of Figs. 2 and 3 . The end of the first Brillouin zone for each direction of propagation is shown in the figure (vertical lines).

high values of $k$ and also at high values of frequency (i.e., at high values of $k_{0} a$ ). These differences correspond to the effect of spatial dispersion, which becomes important when the size of the unit cell is not small in comparison with the wavelength in free space and in the medium. ${ }^{17}$ In such regions, the behavior of the structure is closer to a photonic crystal than to an effective medium. However, inside the lefthanded band (between points marked $2^{\prime}$ and $4^{\prime}$ in Fig. 4), spatial dispersion does not produce significant deviations from isotropy (i.e., the dispersion curves are almost equal for any direction of propagation, as can be seen in Fig. 5). This effect is even more apparent in the frequency band between the unbalanced forbidden band (marked as $3^{\prime}$ in Fig. 4) and the end of the left-handed band (marked as $4^{\prime}$ in Fig. 4).

It is illustrative to compare the band structure of the considered balanced structure with that of an unbalanced one. Thus, Fig. 6 shows the dispersion diagrams for the eigenmodes of two unbalanced structures (which were obtained by varying $t$ in Fig. 1). It can be observed how, as $t$ decreases, the forbidden frequency band increases until the backward-

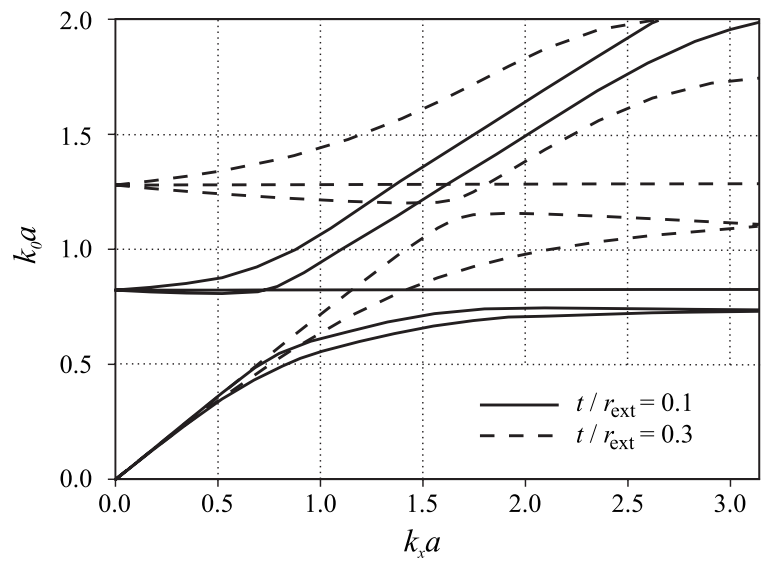

FIG. 6. Dispersion curves along the $\Gamma-X$ direction for the eigenwaves of two unbalanced metamaterials. Parameters are the same as in Fig. 2, except for $t / r_{\text {ext }}$, which is taken as $t / r_{\text {ext }}=0.3$ (dashed lines) and $t / r_{\text {ext }}=0.1$ (solid lines).

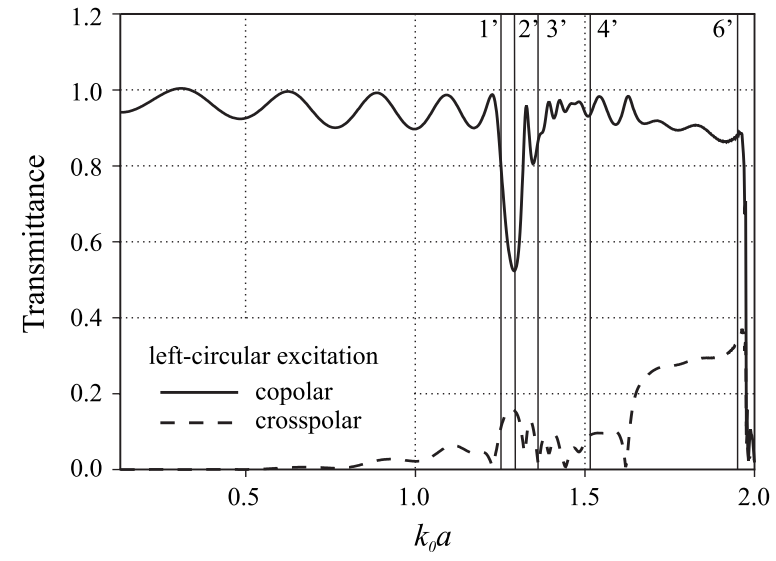

FIG. 7. Simulated transmittance through a seven unit cell thick slab of the metamaterial analyzed in Figs. 2-5 for a left-circularly polarized (LCP) incident wave. Transmittance is plotted for both the co-polar (LCP wave) and the cross-polar (RCP wave) components. Points marked $1^{\prime}-6^{\prime}$ correspond to the marks in Fig. 4.

wave region vanishes, giving a behavior similar to that of a negative- $\mu$ split ring metamaterial. Figure 6 also shows that the tolerance of condition (19) is high. It can be seen that even a change of more than $30 \%$ in the particle thickness does not strongly affect the left-handed passband.

\section{PROPAGATION THROUGH A FINITE SLAB}

In the practical design of a NRI medium, apart from the obvious obtaining of some amount of negative refraction, it should be required that the metamaterial has small reflectance for the beam to be refracted with a significant amplitude. Since for balanced metamaterials it is imposed that $\chi_{e} \simeq \chi_{m}$ in the frequency range of interest, it directly follows from Eq. (6) that $Z(\omega) \simeq Z_{0}$, which ensures a good matching to free space and constitutes an additional relevant advantage of the proposed balanced NRI metamaterials.

In order to illustrate the above feature, the normal incidence of a circularly polarized plane wave on a slab of the proposed chiral medium will be studied through electromagnetic simulations. First, a wave with the same polarization as the left-handed eigenmode of Figs. 4 and 5 is analyzed. The computed transmittance is shown in Fig. 7. It can be seen that the amplitude of the cross-polarized wave is almost negligible, which means that the circular polarization is maintained. The figure also shows that the medium is practically transparent for almost all the considered frequencies, as expected from the aforementioned impedance matching. However, the small ripples observed in the transmittance in Fig. 7 shows that the impedance matching is not perfect. The regions of poor transmittance, between points $1^{\prime}$ and $2^{\prime}$, and beyond $6^{\prime}$, exactly reproduce the corresponding band gaps in Fig. 4. There is also a smaller dip at point $3^{\prime}$, which corresponds to the unbalanced band gap of Fig. 4. Therefore, a useful region of backward-wave propagation ranges from point $3^{\prime}$ to point $4^{\prime}$, which includes the most useful values of $\varepsilon_{r}$ and $\mu_{r}$, as already mentioned.

Backward-wave propagation in the frequency band of interest will be demonstrated by plotting the phases of the 


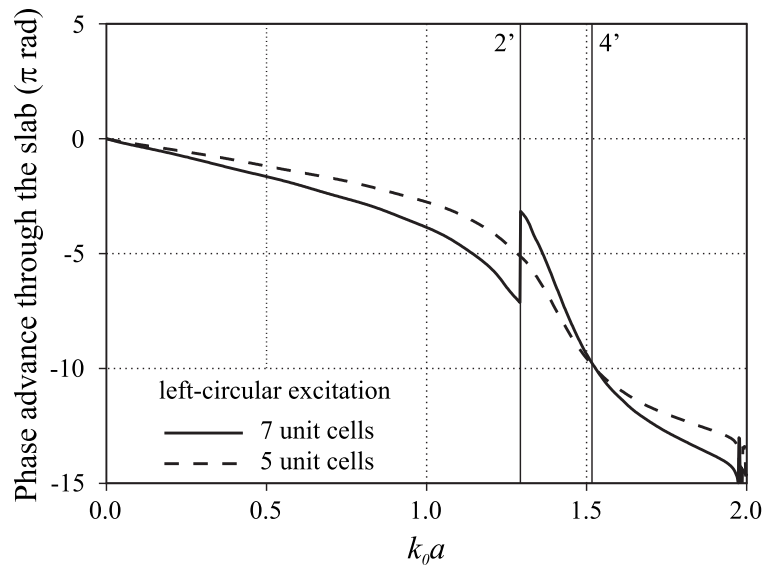

FIG. 8. Phase of the simulated transmission coefficient for the incident LCP wave through a seven unit cell thick slab (solid line) and a five unit cell thick slab (dashed line). Points marked $2^{\prime}-4^{\prime}$ correspond to the marks in Fig. 4.

computed transmission coefficients for two slabs of different thicknesses (see Fig. 8). A zero phase shift between both transmission coefficients was imposed at zero frequency, i.e., for static fields. Also, a convenient phase shift of $4 \pi$ was imposed to the phase of the seven unit cell thick slab at the onset of the left-handed band (i.e., at frequency point $2^{\prime}$ ). This shift is imposed in order to obtain a zero phase shift between both coefficients at point $4^{\prime}$, which corresponds to the zero phase advance point for the backward-wave eigenmode of Fig. 4. As a consequence, the sign of the phase difference between both samples changes from the backward-wave region (between frequency points $2^{\prime}$ and $4^{\prime}$ ) to the forward-wave bands (between zero frequency and $2^{\prime}$, and beyond $4^{\prime}$ ). This result is in complete agreement with the backward-wave propagation postulated for the leftcircularly polarized wave (LCP) between frequency points $2^{\prime}$ and $4^{\prime}$. It should be mentioned that once a zero phase shift is imposed at zero frequency and at the frequency point $4^{\prime}$, the phase jump at $2^{\prime}$ cannot be arbitrarily imposed. Therefore, the fact that the phase jump necessary to fulfill the aforementioned conditions equals to $4 \pi$ provides an additional confirmation of the proposed theory.

Finally, the simulated transmittances for the co-polar and cross-polar componets of an incident RCP wave are shown in Fig. 9. The obtained values for this transmittance are again near unity, except for the band gaps shown in Fig. 4 for this wave, i.e., around frequency point $3^{\prime}$ and beyond $5^{\prime}$.

\section{RACEMIC PERIODIC MEDIUM}

Next, it will be explored the possibility of designing a racemic mixture of chiral SRRs while keeping the necessary symmetries to ensure an isotropic behavior for the metamaterial. For this purpose, it will be considered a simple cubic lattice of periodicity $2 a$ made of cubes as that shown in Fig. 1(a) and another similar cubic lattice made of the same cubes but of opposite handedness. Both cubic lattices can be interleaved, as shown in the inset of Fig. 10, to give a simple cubic lattice whose unit cell is formed by two cubes of op-

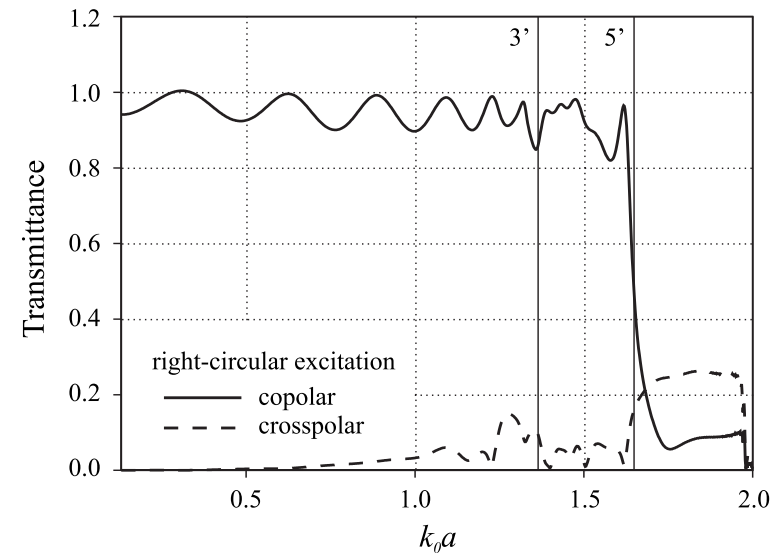

FIG. 9. Simulated transmittance through a seven unit cell thick slab of the metamaterial analyzed in Figs. 2-5 for the co-polar (RCP wave) and the cross-polar (LCP wave) components of an incident RCP eigenmode. Points marked $3^{\prime}$ and $5^{\prime}$ correspond to the marks in Fig. 4.

posite handedness. Since the whole structure is symmetric after inversion (the centers of symmetry are the corners of the cubes), the cross susceptibility $\kappa$ (which is pseudoscalar) must vanish. ${ }^{17}$ Therefore, the whole structure is a balanced isotropic metamaterial with $\kappa=0$. Figure 10 shows the theoretical dispersion relation $\left(k=k_{0} \sqrt{\varepsilon_{r} \mu_{r}}\right)$ of the proposed structure. A frequency band of backward-wave propagation, corresponding to the condition $\varepsilon_{r}, \mu_{r}<0$, can be observed in the figure. Since the phase vector is now simply given by $k$ $=\omega \sqrt{\varepsilon \mu}$, the unbalanced band gap disappears, and the width of the left-handed frequency band decreases in comparison with that of Fig. 3. The wave impedance is also given by Eq. (6). That is, it is almost equal to the free space impedance, which ensures good matching to free space for all angles of incidence. From the above considerations, we feel that these

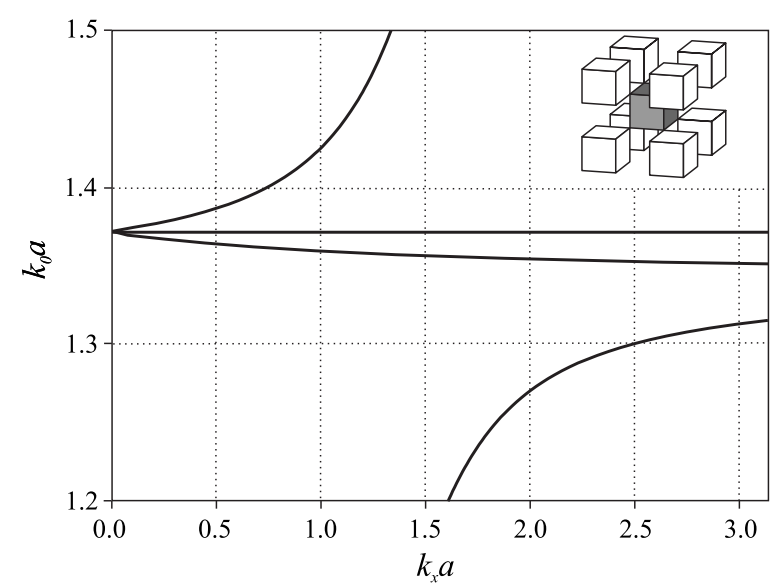

FIG. 10. Theoretical dispersion relation for plane waves propagating in the racemic metamaterial with the simple cubic lattice shown in the inset. Inset: Racemic periodic structure made of two interleaved simple cubic lattices of cubes as that of Fig. 1(a). White cubes are identical to that of Fig. 1(a) and gray cubes are similar cubes made of chiral SRRs of opposite handedness. Chiral SRR parameters are as in Fig. 3 and the periodicity of the structure is $2 a$. The fully degenerate longitudinal mode is now located at $\varepsilon_{r} \mu_{r}=0$. 
racemic isotropic mixtures can be very useful for the design of isotropic regular left-handed metamaterials.

\section{CONCLUSIONS}

Periodic NRI metamaterials based on chiral SRRs have been proposed and demonstrated. An analytical theory has been developed for the design of such metamaterials, which has been validated by careful full-wave electromagnetic simulations. It has been shown that simple cubic lattices of chiral SRRs can provide bi-isotropic NRI metamaterials with a well defined frequency band of backward-wave propagation for one of its plane-wave eigenstates. Transmittance through a slab of finite thickness has been analyzed and good matching to free space has been demonstrated. Besides, a racemic simple cubic lattice of chiral SRRs satisfying the appropriate symmetry has been proposed in order to provide ordinary NRI isotropic left-handed metamaterials with a well defined band of backward-wave propagation for all its planewave eigenstates. Good matching to free space is also expected for these last designs. Besides good matching to free space, the proposed structures have the advantage of being made of a single kind of inclusions, a fact that substantially simplifies their design and fabrication. Applications of the reported concepts in the design of lenses, antennas, and other NRI metamaterial based devices can be envisaged.

\section{ACKNOWLEDGMENTS}

This work has been supported by the Spanish Ministerio de Educación y Ciencia under Projects Nos. TEC200768013-C02-01/TCM and TEC2007-65376/TCM, and by the Spanish Junta de Andalucía under Project Nos. P06-TIC01368 and TIC-253. *1_jelinek@us.es

†marques@us.es

†mesa@us.es

§jdbaenad@unal.edu.co

${ }^{1}$ V. G. Veselago, Sov. Phys. Usp. 47, 509 (1968).

${ }^{2}$ D. R. Smith, W. J. Padilla, D. C. Vier, S. C. Nemat-Nasser, and S. Schultz, Phys. Rev. Lett. 84, 4184 (2000).

${ }^{3}$ D. R. Smith and D. Schurig, Phys. Rev. Lett. 90, 077405 (2003).

${ }^{4}$ T. Koschny, L. Zhang, and C. M. Soukoulis, Phys. Rev. B 71, 121103(R) (2005).

${ }^{5}$ I. Vendik, O. Vendik, I. Kolmarov, and M. Odit, Opto-Electron. Rev. 14, 179 (2006).

${ }^{6}$ A. Grbic and G. V. Eleftheriades, J. Appl. Phys. 98, 043106 (2005).

${ }^{7}$ M. Zedler, C. Caloz, and P. Russer, IEEE Trans. Microwave Theory Tech. 55, 2930 (2007).

${ }^{8}$ C. Monzon and D. W. Forester, Phys. Rev. Lett. 95, 123904 (2005).

${ }^{9}$ J. B. Pendry, Phys. Rev. Lett. 85, 3966 (2000).

${ }^{10} \mathrm{~S}$. Tretyakov, Analytical Modeling in Applied Electromagnetism (Artech House, Norwood, 2003).
${ }^{11}$ S. A. Tretyakov, A. Sihvola, and L. Jylh, Photonics Nanostruct. Fundam. Appl. 3, 107 (2005).

${ }^{12}$ J. B. Pendry, Science 306, 1353 (2004).

${ }^{13}$ R. Marqués, L. Jelinek, and F. Mesa, Microwave Opt. Technol. Lett. 49, 2006 (2006).

${ }^{14}$ J. A. Kong, Electromagnetic Wave Theory (EMW, Cambridge, 2000).

${ }^{15}$ T. G. Mackay, Microwave Opt. Technol. Lett. 45, 120 (2005).

${ }^{16}$ J. D. Baena, L. Jelinek, and R. Marqués, Phys. Rev. B 76, 245115 (2007)

${ }^{17}$ L. D. Landau, E. M. Lifshitz, and L. P. Pitaevskii, Electrodynamics of Continuous Media, 3rd ed. (Pergamon, New York, 1984).

${ }^{18}$ R. Marqués, F. Mesa, L. Jelinek, and J. D. Baena, in Proceedings of Metamaterials 2007, Rome, Italy (Metamorphose VI-AISBL, Louvaine-la-Neuf, Belgium, 2007), p. 214.

${ }^{19}$ R. Marqués, F. Mesa, J. Martel, and F. Medina, IEEE Trans. Antennas Propag. 51, 2572 (2003).

${ }^{20}$ In practical designs, a very low dielectric substrate (foam, for instance) could be introduced between the rings in order to provide mechanical stability and the bridges between them substituted by standard via-hole connections. 